\title{
The Use of Emerging Technologies DloT: Elderly Daily Living in Post-Epidemic Era
}

\author{
Jie $\mathrm{Li}^{1, *}$,Wei Wei Goh ${ }^{2}$, and N.Z Jhanjhi ${ }^{3}$ \\ 123 School of Computing and IT, Taylor's University, Subang Jaya, Selangor, Malaysia
}

\begin{abstract}
As the number of aging population increases, their Quality of Life (QoL) becomes a concern in the society. The elderly is not only vulnerable due to their chronic degeneration issues but may also be insensitive to technological innovation which could possibly improve their QoL in the Industrial Revolution 4.0. The outbreak of COVID-19 has significantly threatened their well-being in their living context and will consequentially change people's perspective towards normal lifestyle after this public health crisis. Recent studies have highlighted the usability of the drone technology in the automation of navigation, monitoring, and load carrying which can potentially facilitate various purposes of use in our daily life. This paper reviews recent academic works related to DroneBased Internet of Things (DIoT) technology and extracts the advantages of DIoT applications, which have the potential to assist elderly's Activity of Daily Living (ADL) in post-epidemic time. Our results suggest that with the low energy consumption, the DIoT techniques are capable of reducing the body exposure under pandemic situation and satisfying the appreciation to normal and digital-connected life in the future. Nevertheless, the limited flying range and low technology penetration among elderly users significantly impede the implementation of DIoT application. Importantly, the DIoT technology upgrades manpower-based manual work. This paper updates to the knowledge of drone technology application in the context of elderly centre during post-pandemic.
\end{abstract}

\section{Introduction}

The advancement of the Internet of Things (IoT) has altered every single facet of daily human activities. Although technology innovation satisfies various aspects of industrialization, it still challenges the enhancement of Quality of Life (QoL) [1] as the main achievable goal in the 4th Industrial Revolution (I.R 4.0) [2]. Under the circumstance of the COVID-19 outbreak, a massive pandemic mortality loss in statistics has caused the apprehension of 198 regions globally [3]. Compared to the younger generations, the elderly faces more critical challenges and has greater fragility rate in Ambient Assisted Living (AAL) context. Although low technology penetration hinders the elderly users for advanced technology adoption due to limited usability [4, 5], affordability [6] and accessibility [7],

\footnotetext{
*Corresponding author: lijie@sd.taylors.edu.my
} 
the situation constantly changes how the elderly embraces the emerging technologies in their daily living [8].

Unmanned Aerial Vehicle (UAV) [9], or drone, has been widely adopted in our daily life when Internet of Things by Drone (DIoT) enables intelligence with full capability and network connectivity in many disciplines. The innovation and diffusion of new civil DIoT technologies assist people in their digital-connected ADLs. This paper aims to review the emerging DIoT innovation and its capabilities to benefit the public in present situation amid the COVID-19 outbreak.

Consequently, the objective of this paper is to reveal the fact that the DIoT innovations are able to be extended into the elderly's AAL whilst functionalizing the ADL in their postepidemic living context. To be specific, the research investigates the advantages and disadvantages of using DIoT for the elderly's daily living in post-pandemic time.

The rest of the paper is arranged as follow: the literature review explains the DIoT innovation, functionalities, and limitations from the related works. In Section 3, we discussed the possibility of DIoT-based technology innovation for the elderly regarding the pandemic crisis and compared the DIoT automation and conventional manpower process. Meanwhile, Section 4 provides the summary while elucidating the pros and cons to have DIoT technologies adopted within the elderly community. We concluded this paper with a proposal of future work in Section 5.

\section{Literature Review}

Most countries have taken the measure of executing the quarantine to control the spread of the Covid-19 pandemic. It is seen as a live saving method while also maintaining social activities with the new norms to avoid panic and reviving the socio-economic aspects of the community. The movement restriction order which has been implemented has dramatically improved the efficiency of pandemic control in many countries [10]. However, the measure of self-isolating has inevitably affected people's mobility for social activities with shortterm loss of freedom and causes economic slowdown in the long run [11]. Nevertheless, DIoT technologies strengthen the capability of UAV in automated surveillance, loadcarrying and specific task performance. These functions enable people to remotely control the devices and reduce body exposure in ADLs and interaction in planned tasks.

\subsection{DloT applications}

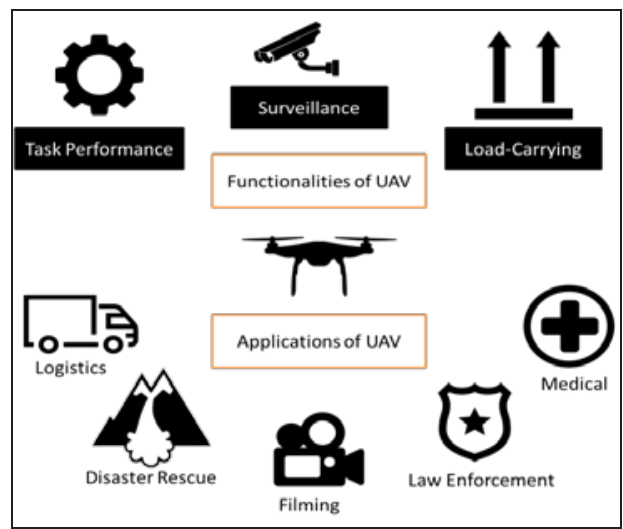

Fig. 1. The Functionalities and Applications of the UAV. 
DIoT depicts the fundamental of layered architecture control for UAV to perform in the different communication environment [12-14]. As shown in Figure 1, UAV has demonstrated its variety of civil usage for daily living purposes such as surveillance, delivery, and task performance in the segments of law enforcement [15], disaster rescue [16], journalism and filming [17], traffic load monitoring [18], and healthcare. In healthcare service, UAV empowers' emergency care [19-21], laboratory testing and organ delivery [22], and disease/injury surveillance [20]. Furthermore, the UAV solution for handling emergency cases accentuates its excellence in real-time monitoring and transportation of medication supplies from many researches [16, 19, 20, 23].

\subsection{UAV movement control}

In term of automated flight, the flight mode of a UAV can be switched in different level of autonomy either by human operator or via on-board CPU in the drone device [21, 23, 24]. To be specific, Proportional integral derivative (PID) mechanism allows the remote control of a drone whereas the Inertial Navigation System (INS) supports the autopilot flight with the parameters of velocity, altitude and position information to define the mission by marking the way point in the close and open routine under multiple modes of rotation and translation. Global Positioning System (GPS) receiver embedded with microcontroller can continuously collect the location information from satellite $[25,26]$, which provides consistent accuracy of the position. GPS/INS-based UAV movement control is a wellplanted UAV system that makes the flying of drone safe and automated.

\subsection{UAV communication protocol}

The UAV communication network is not only about internal flight control module inside of the UAV, but also the involvement of communication channels which synchronize status of the device in a particular system with its corresponding Ground Control Station (GCS) to achieve its purposes of use [27]. In this case, DIoT network demands for the reliability and security of its aerial controlling by means of communicative protocols [28]. There are a few existing challenges and benefits in the implementation UAV protocols.

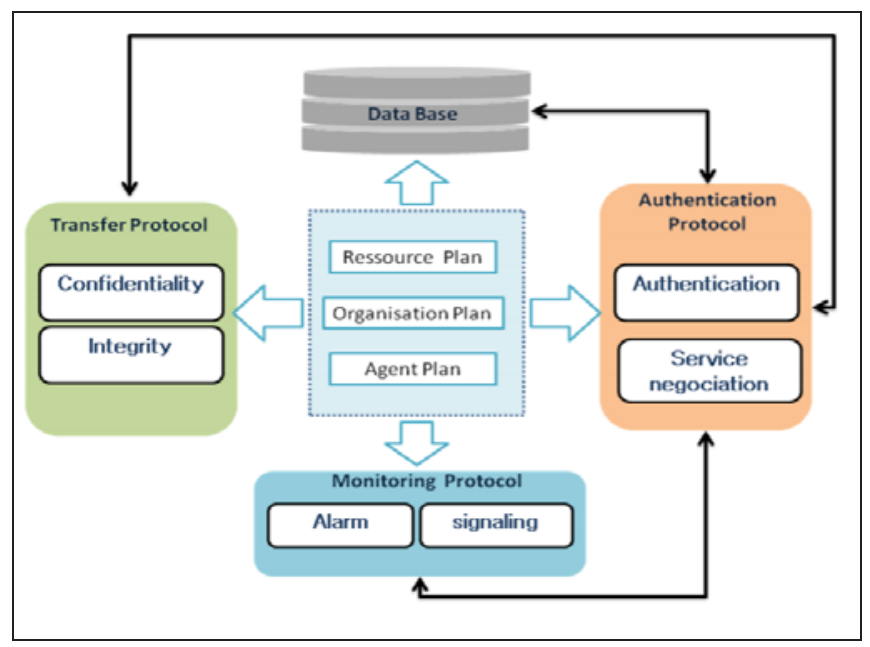

Fig. 2. Security Communication Protocol in UAV and GCS [34]. 
When an UAV contacts with GCS, the station monitors the flight status of the UAV with the data of distance, location, altitude, and status of UAV as well as its components $[27,29]$. In order to achieve a particular task, the GCS is required to establish a highefficient communication with the UAV device via a reliable channel during the remotelycontrolled performance [30]. In that direction, the communication protocol used vitally plays its role in the UAV flight. The most common-used protocol such as UAVCan (Intravehicle communication) [31] and MAVLink (bidirectional UAV-GCS communication) [32, 33] have provided industrial level solution to the UAV remote communication. However, the concerns on the security during the data transmission in the use of UAV, especially, in the security-sensitive applications were never stopped [33]. In this case, the Security Communication Protocol [34] between the UAV and GCS is developed in the combinational structural of transfer, monitoring, and authentication protocols along with the security database to store the log and session keys regarding the high requirements of communication security in UAV applications.

\subsection{UAV routing}

The application of UAV can reduce the cost and time in task performance [35-37]. In the drone-based delivery cases, a successful routing plan supported by mathematics-based computing algorithm (e.g. Dijkstra's) in a Software Defined Network (SDN) can effectively reinforce the logic and performance of application [38]. Dorling et al. [36] exhibits a comprehensive routing algorithm in the input variables (e.g. total cost, time cost, object weight, energy cost, initializing time, routing timing vector, etc.) based on the consideration in the output of delivery efficiency. Furthermore, drone homing is a concept of shortcut navigation for drone flight back to origin. With the support by the visual measuring approach using actual camera, the altitude estimation will be correspondingly computed. Simultaneous localization and mapping (SLAM) approach can solve the issue of location estimation with the variables in an unknown environment through shortcut route plan [37]. With an implementation of an efficient routing algorithm, UAV application can significantly enhance its task performance in different use scenarios. To be summarized, with an implementation of an efficient routing algorithm, UAV application can significantly enhance its task performance in different use scenarios. The application of $\mathrm{UAV}$ can reduce the cost and time in task performance [35-37].

\section{Benefits of UAV for the Elderly}

In this section, we put the insight on how the DIoT system will protect and improve the QoL of the elderly from pandemic threat in the future. Previous studies have demonstrated the capability and system modelling of civil DIoT system. In this case, the comparison between DIoT system and manual work system discovers the pros and cons of DIoT automation adoption in daily use cases.

\subsection{Formatting the text}

In the elderly's living environment, the DIoT techniques are capable of being applied in Surveillance Systems [23, 34], Emergency System [19], and Load-Delivery System [39]. Table 1 indicates numerous UAV applications which are designed to improve the daily norm of elderly people in independent or self-isolating scenario and their QoL with the consideration of digital-connected trend lifestyle in the future. When the independent living 
becomes a necessity for the elderly amid the pandemic outbreak, AAL supports the elderly in their daily routine.

Table 1. The Segments of UAV Application for Elderly Users.

\begin{tabular}{|c|c|c|c|}
\hline System & Objective & Functions & Technique Used \\
\hline $\begin{array}{c}\text { Surveillance } \\
\text { System } \\
{[23,34]}\end{array}$ & $\begin{array}{l}\text { Outdoors } \\
\text { Security }\end{array}$ & $\begin{array}{c}\text { Monitoring } \\
\text { \& Navigation }\end{array}$ & $\begin{array}{c}\text { Image Capture } \\
\text { Analysis } \\
\text { Remote Control } \\
\text { Auto-Pilot } \\
\text { GPS/INS }\end{array}$ \\
\hline $\begin{array}{l}\text { Emergency } \\
\text { System [19] }\end{array}$ & $\begin{array}{l}\text { Outdoors } \\
\text { Emergency } \\
\text { Alert and } \\
\text { First-Aid }\end{array}$ & $\begin{array}{c}\text { Load- } \\
\text { Carrying \& } \\
\text { Navigation }\end{array}$ & $\begin{array}{c}\text { Path-Planning } \\
\text { Delivery } \\
\text { GPS/INS }\end{array}$ \\
\hline $\begin{array}{c}\text { Parcel } \\
\text { Delivery } \\
\text { [39] }\end{array}$ & $\begin{array}{l}\text { Fast \& } \\
\text { Cost- } \\
\text { Effective } \\
\text { Delivery }\end{array}$ & $\begin{array}{c}\text { Load- } \\
\text { Carrying, } \\
\text { Monitoring, } \\
\text { Navigation \& } \\
\text { Communicati } \\
\text { on }\end{array}$ & $\begin{array}{c}\text { Path-Planning } \\
\text { Delivery } \\
\text { GPS/INS } \\
\text { SDN (WSN) } \\
\text { Remote Control }\end{array}$ \\
\hline
\end{tabular}

The implementation of DIoT satisfies the improvement of QoL with the appreciation to normal life after the public health crisis outbreak. First and foremost, DIoT assist the elderly by reinforce their independence of daily living. By using the DIoT technologies, they are able to manipulate the disciplined system for their convenience. For example, the adoption of DIoT-based surveillance system can guard the safety of elderly people in the outdoor context with the support of Telemedicine approach [40, 41]. First-aid kit delivery by drone secures the safety of elderly people once the emergency happens outdoors [39].

\subsection{Less body exposure in ADLs}

Virus may spread through invisible channels from social activities. Nevertheless, the current COVID testing approach, Nucleic Acid Assay, may convey inaccurate information of identifying virus carriers from the crowds of suspicious samples, so such the flaw would cost hidden dangers to others' safety [41]. Unlike manual work process, the automated device is easier to be sterilized for public health and sanitation SOP during the COVID-19. Thus, the system can be utilized as an intermediate entity during the interaction process in ADLs by reinforcing social distancing in absolute segregated interspace.

The concern of infection raises the fear among the elderly and stresses them in their living context with some fear of prompting depression. In comparison to the current approaches used, the DIoT-based technique significantly reduces physical contact in the delivery process and improves hygiene and safety of the elderly people in their ADL amid the public health crisis. For example, the DIoT-based parcel delivery system [34, 35] reduces physical contact in operational process, which is useful to protect people from the dangers of infection and restrict human interaction during a pandemic.

\subsection{Low energy consumption}

The efficiency of future life requires for clean energy which assures sustainable development with low emission and high efficiency in energy use. Electricity, the power source of DIoT systems, has the unprecedented potential in renewable energy (e.g. solar, 
wind, and water) innovation and technology development in the area of Sustainable Energy. There are two significant advantages of DIoT adoption that need to be clarified in this case. The low time and energy consumption system of DIoT represents workload reduction of man-power and utilizes efficient use of sustainable energy.

Table 2. Comparison between the Process of DIoT Automation and Manual Work.

\begin{tabular}{|c|c|c|c|}
\hline & DIoT Automation & Pilot Control & $\begin{array}{c}\text { Man Power } \\
\text { (Conventional) }\end{array}$ \\
\hline $\begin{array}{c}\text { Controlling } \\
\text { Process }\end{array}$ & Automation & Remote Control & Consciousness \\
\hline SOP & Standardized Program & Mixed & Work Guidelines \\
\hline Hygiene Safety & Sterilization & Sterilization & $\begin{array}{c}\text { Hidden infectious danger } \\
\text { Energy Used }\end{array}$ \\
\hline $\begin{array}{c}\text { Distance } \\
\text { Coverage }\end{array}$ & $\begin{array}{c}\text { Less than } 3 \mathrm{~km} \\
\text { circumference }\end{array}$ & $\begin{array}{c}\text { In } 3 \mathrm{~km} \\
\text { circumference } \\
\text { renewable } \& \text { Non- }\end{array}$ \\
\hline
\end{tabular}

The DIoT system such as the drone-based parcel delivery [39] significantly diminishes the cost of time and energy since it is operated in a point-to-point low-altitude flight [37, 41] regardless of road traffics or junctions. Meanwhile, DIoT system enables remote control by operator in short-distance coverage around 3 kilometres circumference, which liberates man-power from the last-mile delivery work. The distance coverage is limited by battery capacity and system power consumption so that the corresponding solutions applied would extend the geographical coverage of DIoT application in the future.

\section{Discussion}

As demonstrated in Table 3, the advantages of DIoT system imply the potential of DIoT techniques for the elderly-centred development and implement in the post-epidemic time. Essentially, the direct routine and homing algorithms with GPS/INS flight control system allows the DIoT system to perform regardless of barriers under both autonomy and pilot control modes. Additionally, the use of renewable and clean energy fulfils the sustainable development goals in global industries. System automation liberates manpower from manual and repetitive works. Lastly, it was noticed that DIoT system has great hygiene sterilization in operation practices.

Table 3. The Advantages and Disadvantages of DIoT in Elderly's Daily Living.

\begin{tabular}{|c|c|}
\hline Advantages & Disadvantages \\
\hline $\begin{array}{ll} & \text { Time reduced direct and Low } \\
& \text { Altitude flight regardless of barriers } \\
\text { - } & \text { Hygiene Safety } \\
\text { - } & \text { Automation of the manpower }\end{array}$ & $\begin{array}{l}\text { - limited coverage circumference } \\
\text { - Low technology penetration }\end{array}$ \\
\hline
\end{tabular}

It needs to be cautioned though that the system's flaws are identified with recommendations. The geographical circumference limits further distance capacity of the DIoT. In order to solve this particular issue in recent years, mainstream researchers and scientists proposed and dedicated to solutions [23, 36, 43], which are the improvement on battery capacity [36, 43] and system efficiency of power consumption [23]. The user acceptance decides the penetration of an innovation technology. Even though, the 
technology penetration among the elderly community is low compared to other groups due to system usability [4], affordability [44, 45], and technophobic users [6], the situation always changes in terms of technology updates in the IR 4.0. With the implementation of UAV applications in elderly's daily living, their digital-connected lifestyle will enhance their QoL even in quarantine during a pandemic crisis.

\section{Conclusion}

The magnitude of public health crisis affects the quality of life in various aspects which include the sustainability of socio-economic development. Though change in lifestyle may not take immediate effect, the public should always be prepared with an alternative to sustain their Activity of Daily Living (ADL) in the emergence of another pandemic outbreak. The expansion of DIoT innovations can potentially improve life especially for the senior citizen. With raising concerns on the pandemic crisis, this study accentuates the importance of DIoT technology adoption among the elderly community to maintain their independence and increase the QoL in their post-epidemic ADLs.

This paper advocates the potential of DIoT innovations in QoL improvement for the elderly. It also reveals the fact that the implementation of DIoT in the senior citizens' daily life is a preferable consideration for less body exposure in social interaction of ADLs. With concerns to the limitations of the study, the authors only focused on the DIoT technology (excluding fixed-wings) adoption among the elderly community rather than other age groups in post-pandemic milieu.

We can conclude that the knowledge obtained inspires emerging innovation of DIoT application not only for the QoL enhancement of the elderly, but also to the rest of population groups in occupying IR 4.0. The innovations could be based on the demands in community services. However, limitations of the current paper calls for further design and development of a DIoT-based medicine delivery system to sustain medication safety of the elderly especially those living independently and at a time of post-pandemic condition.

Our thanks to the Taylor's Internal Research Grant Scheme - Major Funding Scheme (TIRGSMFS/1/2017/SOCIT/011) for the funding and support received for this project.

\section{References}

1. Jing, W., Willis, R., Feng, Z. Factors influencing quality of life of elderly people with dementia and care implications: A systematic review. Archives of Gerontology and Geriatrics, 66(1), 23-41. (2016).

2. Dogaru, L. The Main Goals of the Fourth Industrial Revolution. Renewable Energy Perspectives. $13^{\text {th }}$ International Conference Interdisciplinarity in Engineering 2019. Procedia Manufacturing, Targu Mures, Romania, 46(2020), 397-401. (2020).

3. Meo, S. A., Al-Khlaiwi, T., Usmani, A. M., Meo, A. S., Klonoff, D. C., Hoang, T. D. Biological and epidemiological trends in the prevalence and mortality due to outbreaks of novel coronavirus COVID-19. Journal of King Saud University Science, 32(4), 2495-2499. (2020).

4. Tsirmpas, C., Anastasiou, A., Bountris, P., Koutsouris, D. A New Method for Profile Generation in an Internet of Things Environment: An Application in AmbientAssisted Living. IEEE Internet of Things Journal, 2(6), 471-478. (2015).

5. Brunner, J., Chuang, E., Goldzweig, C., Cain, C. L., Sugar, C., Yano, E. M. Usercentered design to improve clinical decision support in primary care. International Journal of Medical Informatics, 104(May), 56-64. (2017). 
6. Pal, D., Funilkul, S., Vanijja, V., Papasratorn, B. Analyzing the Elderly Users' Adoption of Smart-Home Services. IEEE Access, 6, 51238-51252. (2018).

7. Mitzner, T. L., Savla, J., Boot, W. R., Sharit, J., Charness, N., Czaja, S. J., Rogers, W. A. Technology Adoption by Older Adults: Findings from the PRISM Trial. Gerontologist, 59(1), 34-44. (2019).

8. Hunsaker, A., Hargittai, E. A review of Internet use among older adults. New Media \& Society, 20(10), 3937-3954. (2018).

9. Clark, D. G., Ford, J. D., Tabish, T. What role can unmanned aerial vehicles play in emergency response in the Arctic: A case study from Canada. PLOS ONE, 13(12), 117. (2018).

10. Whitworth, J. COVID-19: a fast evolving pandemic. Transactions of The Royal Society of Tropical Medicine and Hygiene, 114(4), 241. (2020).

11. Nicola, M., Alsafi, Z., Sohrabi, C., Kerwan, A., Al-Jabir, A., Iosifidis, C., ... Agha, R. The socio-economic implications of the coronavirus pandemic (COVID-19): A review. International journal of surgery (London, England), 78, 185-193. (2020).

12. Gharibi, M., Boutaba, R., Waslander, S. L. Internet of Drones. IEEE Access, 4, 1148 1162. (2016).

13. Huang, S., Huang, J., Tang, D., Chen, F. Research on UAV flight performance test method based on dual antenna GPS/INS integrated system. 2018 IEEE 3rd International Conference on Communication and Information Systems, ICCIS, Singapore, 106-116. (2019).

14. Maciel-Pearson, B. G., Akcay, S., Atapour-Abarghouei, A., Holder, C., Breckon, T. P. Multi-Task Regression-Based Learning for Autonomous Unmanned Aerial Vehicle Flight Control Within Unstructured Outdoor Environments. IEEE Robotics and Automation Letters, 4(4), 4116-4123. (2019).

15. Davis, O. Theorizing the advent of weaponized drones as techniques of domestic paramilitary policing. Security Dialogue, 50(4), 344-360. (2019).

16. Ejaz, W., Azam, M. A., Saadat, S., Iqbal, F., Hannan, A. Unmanned Aerial Vehicles enabled IoT Platform for Disaster Management. Energies, 12(14), 2706. (2019).

17. Pugliese, L.D.P., Guerriero, F., Natalizio, E., Zema, N. R. A biobjective formulation for filming sport events problem using drones. Proceedings of the 2017 IEEE 9th International Conference on Intelligent Data Acquisition and Advanced Computing Systems: Technology and Applications, IDAACS 2017, Bucharest, Romania, 2, 639644. (2017).

18. Khan, N. A., Jhanjhi, N. Z., Brohi, S. N., Usmani, R. S. A., Nayyar, A. Smart traffic monitoring system using Unmanned Aerial Vehicles (UAVs). Computer Communications. 157(1), 434-443. (2020).

19. Dayananda, K. R., Gomes, R., Straub, J. An interconnected architecture for an emergency medical response unmanned aerial system. The $36^{\text {th }}$ AIAA/IEEE Digital Avionics Systems Conference, St. Petersburg, Florida, USA, 1-6. (2017).

20. Kumar, G. D., Jeeva, B. Drone ambulance for outdoor sports. Asian Journal of Applied Science and Technology (AJAST), 1(5), 44-49. (2017).

21. Clark, D. G., Ford, J. D., Tabish, T. What role can unmanned aerial vehicles play in emergency response in the Arctic: A case study from Canada. PLoS ONE, 13(12), 117. (2018).

22. Scalea, J. R., Restaino, S., Scassero, M., Blankenship, G., Bartlett, S. T., Wereley, N. An initial investigation of unmanned aircraft systems (UAS) and real-time organ status measurement for transporting human organs. IEEE Journal of Translational Engineering in Health and Medicine, 6(August), 1-7. (2018). 
23. Li, J., Goh, W. W., Jhanjhi, N. Z..A Design of IoT-Based Medicine Case For The Multi-User Medication Management Using Drone In Elderly Centre, in Journal of Engineering Science \& Technology JESTEC. 16(2). (2021)

24. Al Rasyid, M. U. H., Lee, B. H., Sudarsono, A. Wireless body area network for monitoring body temperature, heart beat and oxygen in blood. 2015 International Seminar on Intelligent Technology and Its Applications(ISITIA), Surabaya, Indonesia, 95-98. (2015).

25. Marcelino, I., Laza, R., Domingues, P., Gómez-Meire, S., Fdez-Riverola, F., Pereira, A. Active and assisted living ecosystem for the elderly. Sensors (Switzerland), 18(4), 1-18. (2018).

26. Alves, R. C. A., Gabriel, L. B., De Oliveira, B. T., Margi, C. B., Dos Santos, F. C. L. Assisting physical (Hydro) therapy with wireless sensors networks. IEEE Internet of Things Journal, 2(2), 113-120. (2015).

27. Hong, Y., Fang, J., Tao, Y. Ground control station development for autonomous UAV. In International Conference on Intelligent Robotics and Applications, Berlin, Hidelberg, German, 36-44. (2008).

28. Khan, N. A., Brohi, S. N., Jhanjhi, N. Z. UAV's Applications, Architecture, Security Issues and Attack Scenarios: A Survey. In Intelligent Computing and Innovation on Data Science (pp. 753-760). Springer, Singapore. (2020).

29. Perez, D., Maza, I., Caballero, F., Scarlatti, D., Casado, E., Ollero, A. A ground control station for a multi-UAV surveillance system. Journal of Intelligent \& Robotic Systems, 69(1-4), 119-130. (2013).

30. Lee, W., Lee, J. Y., Lee, J., Kim, K., Yoo, S., Park, S., Kim, H. Ground control system based routing for reliable and efficient multi-drone control system. Applied Sciences, 8(11), 2027. (2018).

31. Khan, N. A., Jhanjhi, N. Z., Brohi, S. N., Nayyar, A. Emerging use of UAV's: secure communication protocol issues and challenges. In Drones in Smart-Cities (pp. 37-55). Elsevier. (2020).

32. Atoev, S., Kwon, K. R., Lee, S. H., Moon, K. S. Data analysis of the MAVLink communication protocol. In 2017 International Conference on Information Science and Communications Technologies (ICISCT), Tashkent, Uzbekistan, 1-3. (2017).

33. Kwon, Y. M., Yu, J., Cho, B. M., Eun, Y., Park, K. J. Empirical analysis of mavlink protocol vulnerability for attacking unmanned aerial vehicles. IEEE Access, 6, 4320343212. (2018).

34. Zouhri, O., Benhadou, S., Medromi, H. A new adaptive security protocol for uav network. International Symposium on Ubiquitous Networking. Morocco, 649657,(2016).

35. Alsamhi, S. H., Ma, O., Ansari, M. S., Almalki, F. A. Survey on Collaborative Smart Drones and Internet of Things for Improving Smartness of Smart Cities. IEEE Access, 7, 128125-128152. (2019).

36. Dorling, K., Heinrichs, J., Messier, G. G., Magierowski, S. Vehicle Routing Problems for Drone Delivery. IEEE Transactions on Systems, Man, and Cybernetics: Systems, 47(1), 70-85. (2017).

37. Bender, D., Koch, W., Cremers, D. Map-based drone homing using shortcuts. IEEE International Conference on Multisensor Fusion and Integration for Intelligent Systems (Mfi), Daegu, Korea, 505-511. (2017).

38. Boychev, I. Z. Research algorithms to optimize the drone route used for security. 2018 IEEE 27th International Scientific Conference Electronics, Sozopol, Bulgaria, 1-4. (2018).

39. Fakhrulddin, S. S., Gharghan, S. K., Al-Naji, A., Chahl, J. An advanced first aid system based on an unmanned aerial vehicles and a wireless body area sensor network 
for elderly persons in outdoor environments. Sensors (Switzerland), 19(13), 2955. (2019).

40. Zaman, N., Ahmad, M. Towards the Evaluation of Authentication Protocols for Mobile Command and Control Unit in Healthcare. Journal of Medical Imaging and Health Informatics, 7(3), 739-742. (2017).

41. Georgi, N., Le Bouquin Jeannes, R. Proposal of a remote monitoring system for elderly health prevention. 2017 International Conference on Smart, Monitored and Controlled Cities, SM2C 2017, Sfax, Tunisia, 69-74. (2017).

42. Pan, Y., Li, X., Yang, G., Fan, J., Tang, Y., Zhao, J., ... Hu, H. Serological immunochromatographic approach in diagnosis with SARS-CoV-2 infected COVID19 patients. Journal of Infection, 81(1), 28-32,(2020).

43. Peng, K., Du, J., Lu, F., Sun, Q., Dong, Y., Zhou, P., Hu, M. A Hybrid Genetic Algorithm on Routing and Scheduling for Vehicle-Assisted Multi-Drone Parcel Delivery. IEEE Access, 7(c), 49191-49200. (2019).

44. Ali, M. A., Alam, K., Taylor, B. Measuring the concentration of information and communication technology infrastructure in Australia: Do affordability and remoteness matter? Socio-Economic Planning Sciences, 70, 100737. (2020).

45. Lee, E. Who are those happy elderly people? Asia-Pacific Journal of Convergent Research Interchange, 4(2), 43-50. (2018). 